Ivchenko D. V., Trufanov I. I. Pelvic fractures in combination with other segmental bone injuries at the polytrauma - possibilities of plateletrich plasma application. Journal of Education, Health and Sport. 2020;10(9):1001-1017. eISSN 2391-8306. DOI http://dx.doi.org/10.12775/JEHS.2020.10.09.119

https://apcz.umk.pl/czasopisma/index.php/JEHS/article/view/JEHS.2020.10.09.119

https://zenodo.org/record/4317542

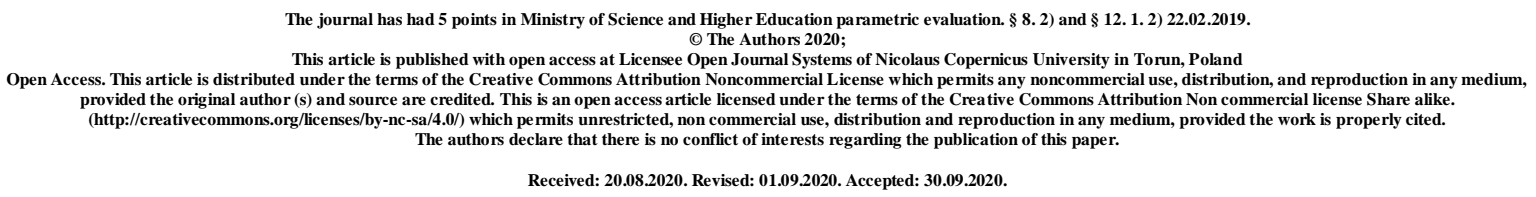

UDC: 616.71-003.93.:577.95]:092-030.52

\title{
PELVIC FRACTURES IN COMBINATION WITH OTHER SEGMENTAL BONE \\ INJURIES AT THE POLYTRAUMA - POSSIBILITIES OF PLATELET-RICH PLASMA APPLICATION
}

\author{
Ivchenko D. V. ${ }^{1}$, Trufanov I. I. ${ }^{2}$ \\ ${ }^{1}$ Zaporozhye State Medical University, \\ Department of traumatology and orthopedics, \\ Maiakovskyi avenue 26, Zaporizhzhia, Ukraine, 69035 \\ ${ }^{2}$ State Institution "Zaporozhye Medical Academy of Postgraduate Education of the \\ Ministry of Health of Ukraine" \\ Department of traumatology and orthopedics, \\ Orikhivske Shosse 10, Zaporizhzhia, Ukraine, 69600
}

\begin{abstract}
Introduction. The urgency of the problem of polytrauma is due to the constant increase in the number of road accidents, armed conflicts, terrorist acts, accompanied by the appearance of a large number of victims with severe and extremely serious injuries.

Purpose of study: to analyze the scientific literature and the results of our own experimental research and to summarize data on current trends and relevance of studying the main factors determining the development of disorders in victims with pelvic fractures in combination with multiple and combined injuries, to improve the prognostic criteria based on a comprehensive
\end{abstract}


assessment of the clinical course of the disease and optimization of treatment by activating reparative regeneration of bone tissue with platelet-rich plasma.

Materials and methods. In the period from 2017 to 2018 in the department of severe concomitant injury of the City Clinical Emergency and Medical Care Hospital of Zaporozhye, 35 patients with pelvic concomitant injury were operated. There were 19 men (54.29\%) and 16 women (45.71\%), aged from 23 to 77 years old, median - 39 years.

22 injured persons (62.86\%) had road traffic injuries, 10 (28.57\%) had household injuries, $2(5.71 \%)$ had production injuries and 1 more $(2.86 \%)$ had others. ISS scale less than 17 points was found in 20 patients (57.14\%), from 17 to 25 - in 12 (34.29\%), from 26 to 40 - in $2(5.71 \%)$, more than 40 - in $1(2.86 \%)$.

In all patients (100\%) varying degrees of pelvic bone injuries were verified. These lesions of the pelvic ring were more often combined with fractures of the proximal femur with a traumatic brain injury - 17 cases $(48.57 \%)$, with concomitant fractures of extremities of other localization - 12 (34.29\%), with intraperitoneal injury - 11 (31.43\%), with vertebral fractures - 9 (25.71\%), with chest trauma - 6 (17.14\%).

Results. In the main group of patients with treatment by platelet-rich plasma radiological visible adhesions of the key injured area in 8 weeks occurred in 10 people (83.33\%). In 1 patient $(8.3 \%)$ fusion occurred after 12 weeks and in $1(8.3 \%)$ - 16 weeks after surgery. In the control group fusion at 8 weeks was detected in 14 patients (60.87\%), in 8 patients $(34.78 \%)$ - at 16 weeks.

Conclusion. Reliably substantiated data were obtained according to the results of the analysis of contingency tables $4 * 4$ and the calculation of $\chi^{2}$ for the efficiency coefficient according to the Wald formula (Chi-square 6.87 with $\mathrm{p}=0.009$ ), which indicate the best clinical positiveness of the technique with the additional introduction of platelet-rich plasma into the treatment regimen as part of assessing the dynamics of fracture consolidation processes in victims with multiple and concomitant injuries, which allows us to predict a favorable outcome in these patients.

Key words: polytrauma; platelet-rich plasma; reparative regeneration; osteogenesis; bone remodeling; pelvic fractures.

Revalence. The urgency of the problem of polytrauma is due to the constant increase in the number of road accidents, armed conflicts, terrorist acts, accompanied by the appearance of a large number of victims with severe and extremely serious injuries. Severe injuries are one of the 
three main causes of death, and in the category of people under the age of 40 , this cause comes first, and among adolescents and young people, this figure reaches $80 \%$ [1].

Accident injuries dominate all over the world in the category of injuries. According to the United Nations, more than 1.25 million people die each year in road accidents worldwide [2]. Today, for the whole of civilized society, severe trauma is the main cause of death, as mostly the youngest and able-bodied part of the population dies from injuries. Assessing the damage from injuries, it should be noted that the number of unlived years, they are much higher than those of cardiovascular, cancer and infectious diseases combined [3]. All of the above has been a powerful impetus for the development of traumatology, surgery, neurosurgery, resuscitation and other fields of medicine that study the problem of injury. In the category of injuries, the complexity of the existing problems, mortality and disability are dominated by severe combined trauma and its most severe form - polytrauma.

There are the highest mortality and disability in this group of victims that we can observed. The frequency of infectious complications with polytrauma, according to various authors, is from 70 to $98 \%$; among them, a high proportion are severe infectious processes, primarily generalized [4]. Complications of polytrauma include traumatic shock, hemo- and pneumothorax, nosocomial pneumonia, fat embolism, thromboembolism, venous thrombosis, brain contusion, brain dislocation, intestinal atony. Victims with polytrauma are characterized by the simultaneous development of tissue disorders that trigger inflammatory reactions. Due to the extremely large amount of antigens, especially in the presence of traumatic shock, patients with polytrauma naturally have a decrease in immunological reactivity in the acute period of traumatic illness. In the post-shock period, when exogenous and endogenous bacterial invasion are present as a pathogenetic factor and comes to the fore, the lack of immune protection leads to inflammatory and purulent-septic complications [5].

According to some authors, if $17 \%$ of patients die in first aid 30 minutes after injury, then mortality increases 2 times in 45 minutes. The critical period of first aid, after which mortality increases sharply, is 3 hours [6]. Surgical tactics, based on an objective assessment of the severity of the condition and new technologies for diagnosis and treatment, can reduce mortality by $14.3 \%$, reduce the incidence of complications by 2 times; reduce the average duration of inpatient treatment and length of stay in the intensive care unit by 1.5-2 times [7]. Objective assessment of the severity of injuries is a necessary tool for deciding on the nature of surgical treatment and intensive care, the optimal place of treatment, to compare the results depending on the methods of treatment and the quality of care. 
Purpose of study: to analyze the scientific literature and the results of our own experimental research and to summarize data on current trends and relevance of studying the main factors determining the development of disorders in victims with pelvic fractures in combination with multiple and combined injuries, to improve the prognostic criteria based on a comprehensive assessment of the clinical course of the disease and optimization of treatment by activating reparative regeneration of bone tissue with platelet-rich plasma (PRP).

Materials and methods. The effectiveness of treatment depends on the assessment of the severity of the condition of the victims and the qualifications of the surgeon / traumatologist, in everyday clinical practice is based on personal medical experience, knowledge and intuition. Such an assessment is very subjective and often because it is not accurate or erroneous. Due to the use of a highly effective system and scale for assessing the severity of injuries in victims are relevant for optimizing and objectifying the patient's condition and prognosis.

Currently, there are more than 50 scales in the world to assess the severity of injuries and the severity of patients with severe mechanical trauma. According to the results of researchers, depending on the task set in creating this international scale, they can be divided into three types. The first type of scale is designed to sort the victims at the scene (Trauma Index - TI; Illness Injury Severity Index - IISI; Triage Score - TS); the second is to retrospectively assess the severity of the injury for further analysis and planning (Trauma and Injury Severity Score TRISS; A Severity Characterizarion of Trauma - ASCOT) and the third type - for prognostic assessment of patient severity and treatment outcomes in intensive care units (Acute Physiology and Chronic Health Evaluation - APACHE I, II, III; Simplified Acute Physiology Score - SAPS; Multiple Organ Dysfunction Scene - MODS; Therapeutic Intervention Scoring System - ISS).

The main tasks of complete and urgent diagnosis at the hospital stage are: in case of skull and brain injuries - neurological examination, radiography, lumbar puncture, echoencephalography, electroencephalography, overlay search milling holes; in case of chest injuries - physical examination, radiography, puncture of the pleural cavity, in case of intense pneumothorax and hemothorax - drainage of the pleural cavities; at abdominal injuries - general clinical examination, laparocentesis, prolonged catheterization of an abdominal cavity, laparoscopy, radiography; in pelvic injuries - a thorough clinical examination, catheterization of the bladder, radiography, cysto- or ureterography, hourly monitoring of diuresis; in case of limb injuries - a thorough examination of all areas to exclude damage to internal organs, detailed examination of the injured segment, radiography, according to the indicators (ischemia) - a special examination of blood vessels (angiography). In parallel with the diagnostic stage, anesthesiology and resuscitation care is performed, which includes anti-shock therapy. 
In the period from 2017 to 2018 in the department of severe concomitant injury of the City Clinical Emergency and Medical Care Hospital of Zaporozhye, 35 patients with pelvic concomitant injury were operated. There were 19 men (54.29\%) and 16 women (45.71\%), aged from 23 to 77 years old, median - 39 years.

22 injured persons (62.86\%) had road traffic injuries, 10 (28.57\%) had household injuries, $2(5.71 \%)$ had production injuries and 1 more $(2.86 \%)$ had others. ISS scale less than 17 points was found in 20 patients (57.14\%), from 17 to 25 - in 12 (34.29\%), from 26 to 40 - in $2(5.71 \%)$, more than 40 - in $1(2.86 \%)$.

In all patients (100\%) varying degrees of pelvic bone injuries were verified. These lesions of the pelvic ring were more often combined with fractures of the proximal femur with a traumatic brain injury -17 cases $(48.57 \%)$, with concomitant fractures of extremities of other localization - 12 (34.29\%), with intraperitoneal injury - 11 (31.43\%), with vertebral fractures - 9 (25.71\%), with chest trauma $-6(17.14 \%)$.

Depending on the method of treatment, patients were divided by random numbers into 2 groups: group 1 (control group) included 23 patients with polytrauma, in the treatment of which used standard therapies. Group 2 (main group) consisted of 12 patients with polytrauma, in the treatment of which platelet-rich plasma was also used in addition to standard methods. The groups were comparable by clinicals, demographics, gender signs, by severity and groups of damage. Surgical tactics and the volume of surgical interventions on the musculoskeletal system were performed in accordance with routine protocols and personal selection of the treatment in both groups.

In the acute period, emergency operations related to the dominant combined injury were performed, as well as bone stabilization was performed in open fractures and unstable fractures of the pelvic bones. On admission, in most cases, patients underwent skeletal traction. After stabilization of the condition, one or another type of osteosynthesis were performed in the period from the first day to 3 weeks after injury. When performing operations, both standard techniques described in the methodical recommendations and original configurations of external fixation devices, as well as reconstructive osteosynthesis with plates with angular stability of screws were used. Regardless of the method of surgical treatment, the patients became more active the day after the operation. They learned to get out of bed on their own, stand up, then began to move with crutches or other aids. Then, for 1.5-2 months, the victims went to full load. In patients with external fixation devices, in addition, twice a day conducted physical therapy classes, with the implementation of passive and active movements in all joints of the injured limb. The groups evaluated the frequency of postoperative local and somatic complications, the length of hospital 
stay at the initial treatment, the number of re-hospitalizations associated with the correction of the treatment process, long-term treatment results. In studying the latter, we used the MathisLuboszyc-Schwarzberg evaluation system. Pain, radiological signs of adhesions, segment shortening, segment deformation, range of motion in adjacent joints, soft tissue atrophy, vascular and neurological disorders, purulent complications were evaluated in points. The overall assessment of the treatment outcome was obtained by summing the numerical values in points of all indicators to the total number of indicators. Anatomical and functional result was considered good, in which the treatment index was 3.5-4 points, satisfactory - 2.6-3.4 points, unsatisfactory 2.5 points or less.

In the treatment of patients of group 2 was used PRP - the concentration of biologically active molecules - growth factors in plasma obtained from the patient's own blood. The blood syringe is placed in the socket of the centrifuge rotor, in the opposite socket - a counterweight or a second syringe of equal volume. Centrifugation is carried out for 5 minutes at $1500 \mathrm{rpm}$. The syringe is then drawn upright so that the plasma does not mix with the precipitated erythrocytes. To transfer 3-5 $\mathrm{ml}$ of plasma from the large outer syringe to the small inner syringe, slowly push down on the outer syringe while slowly pulling up the plunger of the small inner syringe. The small inner syringe is unscrewed, the needle is put on, after which the plasma will be ready for use - injection. When used in conjunction with an anticoagulant, use plasma up to 4 hours after blood collection.

The ISS scale was used to assess the severity of the injury and the condition of patients in the initial and early periods of traumatic illness. The effectiveness of surgical interventions was determined at three degrees of severity of the patient's condition by calculating the coefficient of efficiency of operations in points $\mathrm{K}$ (according to Wald's formula). At the stages of surgical interventions, the dynamics of the clinical condition of patients was assessed in comparison with the initial one. Changes in the condition of the victims were interpreted by us as favorable (the patient's condition improved by all objective indicators) or unfavorable result (respectively, there was a negative trend in the coming days after surgery).

The distribution normality analysis was evaluated according to the preferred Shapiro-Wilk (W) criteria. When it was impossible to reject the null hypothesis of statistically significant differences in the distribution of variables from normal, nonparametric data analysis methods were used, and in other cases, parametric methods. In the case of a distribution other than normal or analysis of ordinal variables, the Mann-Whitney U-test were used for 2 unrelated samples. Comparison of the two groups on the background of treatment was performed using the Wilcoxon test. Comparison of groups on a qualitative basis was performed using $\chi^{2}$ in the analysis of 
conjugation tables. The results of the study were processed using the statistical package of the licensed program "STATISTICA ${ }^{\circledR}$ for Windows 6.1" (StatSoftInc., № RGXR412D674002FWC7), as well as "SPSS 17.0", "Microsoft Excel 2016". Some statistical procedures and algorithms are implemented in the form of specially written macros in the appropriate programs. Differences at $\mathrm{p}<0.05$ were considered statistically significant for all types of analysis.

Results and discussion. In the main group of patients treated with PRP, radiologically visible adhesion of the key injured area after 8 weeks occurred in 10 people (83.33\%). In 1 patient $(8.3 \%)$ the adhesion occurred after 12 , and in another $(8.3 \%)$ - 16 weeks after surgery. In the control group, adhesions at 8 weeks were detected in 14 patients $(60.87 \%)$, in $8(34.78 \%)-$ at 16 weeks. The postoperative course in 1 patient (4.35\%) was complicated by the lack of adhesion after 16 weeks and the presence of a cavity of bone resorption. In this patient, bone autoplasty was not used due to the unsatisfactory condition of the fragments.

Also, it is reliably substantiated according to the results of the analysis of conjugation tables $4 * 4$ and calculation of $\chi^{2}$ for the efficiency coefficient according to the Wald formula (Chi-square $=6.87$ at $\mathrm{p}=0.009$ ), to show the best clinical positivity of the technique with additional introduction into the treatment of PRP concentrate in the assessment of the dynamics of fracture consolidation processes in victims with multiple and combined injuries, which in the complex, allows to predict a favorable outcome.

Splicing control was performed mainly by radiography, but the sensitivity of this method is relatively low. The presence of organized bone beams between the fragments can be recognized at an earlier date during computed tomography (CT), which allows you to start rehabilitation earlier. The number of unsatisfactory results of treatment with the use of free or vascularized bone grafting, and without it indicates the depletion of osteoinductive and reparative bone reserves. One of the factors of treatment should be the stimulation of these processes, which is facilitated by a number of active substances contained in PRP.

Recently, research has been conducted on the use of natural biologically active agents in various fields of medicine. The study of the properties and possibilities of application of PRP belongs to this group of scientific research. The most available source of autogenous growth factors are platelets, as their alpha granules contain various growth factors, such as platelet growth factor (PDGF-aa, PDGF-bb, PDGF-ab), two transforming growth factors (TGF-b1, TGF-b2), epithelial growth factor (EGF), insulin-like growth factor (IGF), fibroblast growth factor (FGF), antiheparin factor, platelet activating factor. Conducted by number of researchers [8], molecular studies of cell proliferation, migration and immunoblotting in keratinocytes treated with PRP 
showed a significant increase in cell proliferation, which led to a significant acceleration of regenerative processes.

In 1965 Marshall R. Urist of the University of California first used PRP to stimulate osteogenesis. He proceeded from the results obtained by his predecessors, which indicated both the pronounced angiogenic and osteogenic activity of substances contained in platelet granules. These substances (platelet-derived growth factor (PDGF), transforming growth factor-p1 (TGFp1) and transforming growth factor-p2 (TGF-P2)) of polypeptide nature belong to the group of growth factors and along with epidermal growth factor, insulin and others factors characterized by a high degree of affinity and a relatively long time of primary exposure to progenitor cells (5-7 days). The use of PRP to stimulate osteogenesis in the replacement of defects of the bones of the facial skull has been widely used in maxillofacial surgery [9]. Comparative evaluation of bone maturity showed that when using PRP and bone material, the bone matured in 1.62-2.16 times faster than when using the same bone material without PRP. With increasing bone regeneration, the duration and cost of treatment naturally decreased.

Terminologically correct it is considered to speak about PRP at concentration of thrombocytes from 700 thousand to 1 million in $1 \mathrm{mkl}$ of plasma, and at the minimum content in it of erythrocytes. According to the latest scientific data, to optimize the processes of regeneration in the wound requires a platelet concentration of at least 1 million in mkl. Obtaining such a concentration is a necessary condition for the effectiveness of the method. It was also found that the required platelet concentration is unattainable with a single centrifugation of blood, regardless of the centrifugation time and speed. In addition to the high concentration of platelets, the PRP must have acceptable manipulative properties that allow it to be mixed with various osteotropic materials. Elevated white blood cell counts, according to some authors, are also desirable. In addition, several other necessary conditions must be met. This is the sterility of the material and morphologically complete platelets. The relationship between the clinical properties of PRP with the content of a large number of platelets in it is confirmed in many studies. Their high concentration in the wound provides a large number of growth factors and is a necessary condition for both effective hemostasis and for a favorable course of the reparative process.

The role of platelets in the wound is not limited to the blood clotting reaction. About 20 biologically active substances contained in the alpha granules of platelets are released when the latter enter the wound. Most of these substances are growth factors that accelerate the healing process. Among them: PDGF - platelet-derived growth factor, TGF- $\beta$ - transforming growth factor beta, TGF- $\alpha$ - transforming growth factor alpha, EGF - epithelial growth factor, FGF fibroblast growth factor, interleukin-1. Currently, there are several methods of obtaining PRP. 
They are all based on the fractional separation of venous blood by centrifugation. Several types of centrifuges are proposed, specially designed for obtaining PRP. The most famous system is SmartPRP, from the American company Harvest. Obtaining PRP in this case is carried out in two stages. The first stage involves centrifuging the blood at a speed of $3600 \mathrm{rpm}$. At the same time there is a separation of erythrocytes from plasma with other elements. The second is centrifugation at a speed of $3000 \mathrm{rpm}$ to form the clot itself. Recently, the method of obtaining PRP by means of a single centrifugation is often used. The laboratory centrifuge supporting work at a speed from 2800 to $3000 \mathrm{rpm}$ is used. Another component of this system are $9 \mathrm{ml}$ tubes containing coagulation activator $(\mathrm{SiO})$. The advantages of the technique are relative cheapness, speed of receipt (not more than $10 \mathrm{~min}$ ), a small amount of blood required $(9 \mathrm{ml})$ to obtain a minimum amount of PRP (about $3 \mathrm{ml}$ ) [10].

Thus, modern methods of obtaining PRP are quite perfect, they allow you to quickly get the required amount of material directly in the operating room. Each of them offers approximately equal quality of material, and they differ only in a set of delivery and convenience in work with them.

The first mentions of local use of PRP are found in the publications, where concluded the feasibility of making fibrin gel from the patient's blood, which is lost during surgery. At that time, the term PRP was not yet used. Only later it became clear that the useful properties of this substrate are due to the high concentration of platelets. Animal experiments were performed to confirm the effectiveness of the use of PRP. There were created in the experiment in horses various surgical wounds and applied to their surface PRP [11]. Then performed a macroscopic assessment of the wound process, as well as histological and cytological examinations. When using PRP there was an intensive formation of collagen fibers, the wound surface at an earlier date was covered with morphologically complete epithelium.

It was noted that under the influence of growth factor improves bone remodeling, including improving the structure of bone tissue and its mineralization [12]. Bone growth factors reduce the inflammatory response and promote healing of injured bone during osteotomy and its mineralization. Also noted the best reconstruction of artificial bone - hydroxyapatite, tricalcium phosphate. It is believed that the properties of growth stimulants have members of a large family, which includes various types of bone morphogenetic protein (BMP). A distinctive feature of the individual components of this family is the phenomenon of osteoinduction. The family of growth factors - transforming growth factor P (TGF-P), is 30-40\% of the amino acid. These include extracellular molecules (morphogens). They reproduce the same cycle as the direct development 
of the bone itself. BMP was detected in embryonesis, in the treatment of fractures and in tissue preparations of osteosarcoma.

TGF-P was found in the same superfamily cooperation as BMP. It is present in the same phases of bone healing. In inflammation, platelets are released, and in turn TGF- $\beta$ is released from them when mesenchymal cells are formed. Chemotaxis is required for bone formation. Studies [13] have established the functionality of PRP in bone grafting - stimulation of osteogenesis. Under the influence of growth factors from the bone matrix as a result of protein synthesis was the formation of bone cells. This method began to develop in the early 90's of last century, but received its development in the clinic only after 8-10 years.

Phylogenetic analysis of PRP has established its capabilities in the regeneration of various tissues. In cell culture, it stimulated their growth and acted as an anabolic parathyroid hormone. A number of authors noted that PRP in the clinic cooperates well not only with autogenic, but also with xenogeneic and synthetic tissues, including tricalcium phosphate "Cerasorb". In addition, it was noted that the use of PRP in patients improved the function of the liver, heart, endocrine organs, as well as general health.

The experiment established the stimulation of osteoblasts under the influence of bone growth stimulants - TGF-p \FGF, BMP 2 and 4. Subsequently, this phenomenon was confirmed in vivo [14]. There were detected acceleration of tissue healing under the influence of PRP, which stimulates osteogenesis and limits osteoclastic bone resorption. This leads to the restoration of blood supply. At the stage of TGF osteogenesis, the number of osteoblast cells increases, their function is regulated and bone matrix synthesis increases. The researchers noted that there is inhibition of the synthesis of type II collagen, but stimulates the development of type I collagen. In the process of bone reconstruction, TGF helps to build bone and improve its quality.

It is known that conservative treatment in group of patients with bone fracture and osteoporosis rarely gives a positive effect. As a rule, various methods of autoplasty are used, the main purpose of which is to stimulate reparative osteogenesis, which in $76 \%$ of cases [15] leads to recovery. However, in almost a quarter of patients the desired result is not achieved, which leads to repeated rheosteosynthesis operations, and sometimes to amputation of limbs at different levels, especially in the case of infected false joints.

Thus, in this study by a group of authors, all patients in the main group are patients who have already undergone a number of standard surgeries for pseudoarthrosis, unfortunately without effect. And, as a rule, researchers used in these cases treatment of PRP as the last chance to help the patient. In the process of accumulation of certain experience by the authors, they have 
empirically developed indications for the use of this technique, which they hope to optimize after the end of research [16].

Hypertrophic and, especially, hypotrophic pseudoarthrosis are also, according to researchers, an indication for the use of PRP, in some cases supplemented by a small amount of autograft as a "focus of regeneration". Patients with homeostatic changes of somatic origin also need to use PRP to reduce the duration of operating time and to stimulate reparative processes.

Given all the above, as well as the practical impossibility of using high quality xenoplastic materials and modern fixators in the existing socio-economic conditions, the use of PRP as a highly effective environment to optimize the process of regenerative repair is shown to almost all patients with osteogenesis complications such as pseudoarthrosis.

The presence in the patient of infected nonunion or pseudoarthrosis complicated by chronic osteomyelitis in remission is an indication for the use of PRP, saturated with antibiotics, according to the sensitivity of the microbial flora, or a wide range of action. The authors consider allergic reactions to $\mathrm{CaCl}_{2}$ and thrombin to be an absolute contraindication to the use of PRP, but such complications were not encountered in the operated patients of this study [17].

The relative contraindication probably includes confirmed thrombocytosis, but this is a rather rare pathology. Osteomyelitis in the acute stage is also a contraindication for PRP.

The following authors studied [18] the effect on the regeneration process after osteotomy of the ribs of recipients (laboratory rats) of suspensions of gel tizol and lyophilisate rich in PRP of intact donors in the composition of means for external use. The control group of animals was applied a similar dilution without PRP. The study of the cellular component of the regeneration region and the biochemical parameters of the blood of the recipients were performed 10 days after surgery. In the regenerate tissues of the experimental animals group there were detected a larger number of cells of the osteoblastic differentiation line and less - chondroblastic differentiation line $(441 \%$ and $45 \%$ of the control values, respectively). In the serum of animals of the experimental group were found a lower concentration of calcium (79\% of control values). The authors concluded that the use in the treatment PRP in experimental group of animals accelerates regenerative processes.

The following article [19] presents a clinical example of the use of PRP in the treatment of a 37-year-old patient with avascular necrosis of the left femoral head at the stage of fragmentation (4B according to the ARCO classification). PRP was obtained by centrifugation for $5 \mathrm{~min}$ at a speed of $1500 \mathrm{rpm} 15 \mathrm{ml}$ of autologous whole venous blood taken in a special two-sided syringe. Immediately before the use of small bone grafts, the volume of PRP was mixed and tightly packed in the defect of the femoral head, formed after removal of necrotic tissue. 0.3-0.4 ml of 
PRP was left in the syringe and injected into the graft-replaced necrosis zone. 6 months after the operation on the images of CT transitions and radiographs of the pelvis of the patient showed signs of reorganization of bone tissue, preserved sphericity of the femoral head. The intensity of pain on the visual-analog scale, the number of points on the Harris scale and the scale for the assessment of disorders in osteoarthritis of the hip (HOOS) before treatment were $60 \mathrm{~mm}, 45$ and 33 points, respectively, after treatment $-10 \mathrm{~mm}, 78$ and 78 points. Thus, the replacement of the area of the focus of avascular necrosis of grafts in combination with PRP during organ-sparing operations allows to delay operations of total hip arthroplasty not only in the early but also in the later stages of the disease.

The authors conducted an experiment [20] on 26 outbred dogs of both sexes aged $1.5 \pm$ 0.09 years with a body weight of $17 \pm 0.5 \mathrm{~kg}$. After transverse osteotomy of the acetabulum $(\mathrm{n}=$ 20) or the body of the acetabulum $(n=6)$, followed by repositioning and stable external fixation of the fragments, all animals from the 2 nd to the 5 th day of the postoperative period underwent intra-articular or intraosseous round-the-clock administration of saline ( $0.4 \mathrm{ml} / \mathrm{day})$. Animals of the experimental series were additionally injected 1 time / day with $1 \mathrm{ml}$ of a composition consisting of autologous blood plasma in combination with solutions of ascorbic acid and glucose. The device was dismantled on the $21 \mathrm{st}$ day of the experiment. Animals were removed from the experiment on the 14th and 42nd day after surgery. As a result of this study in control animals (intravascular and intradermal injection of saline) formed a fibrocartilage connection of the pelvic fragments, and after dismantling the device was deformed bone due to secondary displacement. In the experimental groups, the primary bone connection of the fragments was observed on the 14th day of the experiment; after dismantling the device, bone consolidation is preserved. Autologous blood plasma in combination with metabolically active substances ascorbic acid and glucose had a pronounced stimulating effect on the fusion of pelvic fractures with its local introduction in the early post-traumatic period. The authors believe that the developed method of stimulating reparative osteogenesis is quite minimally invasive, allows early functional load, allows you to manage the reparative process, which is based on the biological principles of tissue regeneration.

In 92 white female rats [21] of self-breeding, a group of researchers studied the effect on the process of fusion of tibial fractures, as well as the exchange of $\mathrm{Ca}^{2+}$ ions, phosphorus and $\alpha$ phosphatase activity in blood plasma. The process of fracture fusion was identical in duration of groups II and III, in which PRP was introduced into the fracture site and phonophoresis was performed with a fixed dose of venom. The groups differed in the nature of the process of callus formation. So in groups I and III the process occurs on osteogenic type, and in group II on chondrogenic. When using PRP, alkaline plasma phosphatase is activated and the content of 
phosphorus and calcium in the blood is reduced. Under the influence of phonophoresis, the $\mathrm{Ca}^{2+}$ access to the fracture zone is activated, but in contrast to group II, this process is temporary. The authors note, first of all, that the PRP and phonophoresis with Apizartron promote more active inflow of calcium into the fracture area, as well as promote the activation of repair processes in the osteocyte population.

The authors in the study treated 24 patients with severe gunshot wounds of the extremities with fractures of the C1-C3 group (according to the universal classification AO / ASIF) for 4 months [22]. 11 patients of the main group in addition to conventional methods were treated using vacuum aspiration device HEACO FORYO to clean the wound and the introduction of PRP factor according to ARTHREX to stimulate local reparative processes, 13 patients of group 2 were treated by conventional methods, antibacterial therapy, surgery. Compared with the control group, in the main group the duration of treatment decreased by $8 \pm 2.8$ days, the number of complications by $33 \%$, which allows us to recommend the introduction of PRP by the method of choice in the treatment of the above pathology.

According to the literature [23], the frequency of fractures that have not fused and require additional surgical treatment is from 12.5 to $26 \%$. The aim of these authors was to study the clinical-radiological and morpho-mechanical features of the regenerate in patients with unfused diaphyseal fractures, to justify the choice of treatment and methods of stimulating bone regeneration. The researchers presented the experience of treating 73 patients with non-fused diaphyseal fractures of the extremities. Among them, clinical groups were identified on the basis of assessing the deformity of the regenerate. The indications for the use of closed compression osteosynthesis, as well as various types of plastic materials (small spongy autografts alone and in combination with PRP, autofibrin gel and other drugs containing fibrin) have been clarified. Author's external fixation devices were used to connect the bone fragments, which functionally loaded the limb. Positive results were obtained in $95 \%$ of cases when using PRP.

Thus, all the above indicates the prospects and extreme medical and social relevance of developing new minimally invasive methods of surgical treatment of patients with pelvic fractures with combined multiple injuries of the skeletal system with the use of available and effective stimulators of osteogenesis, especially such promising as PRP in order to reduce the period of incapacity for work and further rehabilitation. Given all the information available in literature on the high efficiency of PRP in various fields of medicine and the lack of clear systematic information on the use of PRP in various cases of polytrauma, it is considered extremely important and necessary to conduct appropriate research and summarize the data for further clinical use. 
Conclusions. 1 . The analysis of the available modern literature testifies to high urgency of search of new innovative methods of treatment at the combined and multiple trauma of the musculoskeletal system.

2. To date, is not enough substantiated clinical studies on the method of additional administration of autologous plasma in combined injuries of the pelvic bones and other structures of the musculoskeletal system, in contrast to isolated lesions and degenerative diseases.

3. Reliable data were obtained, according to the results of the analysis of conjugacy tables $4 * 4$ and calculation of the $\chi 2$ index for the efficiency coefficient according to Wald's formula (Chi-square 6.87 at $\mathrm{p}=0.009$ ), which indicate the best clinical positivity of the method with additional introduction into the scheme of treatment of PRP in the framework of assessing the dynamics of fracture consolidation processes in victims with multiple and combined injuries, which in the complex allows to predict a favorable outcome in these patients.

4. Thus, it was shown a statistically significant effect on the prognosis and effectiveness of treatment of patients with pelvic fractures on the background of polytrauma compared with routine treatment protocols, which also requires further observations and studies to assess the safety of this method in the practice of traumatologists.

5. The use of PRP for bone regeneration, along with traditional methods of surgical tactics and the standard volume of surgical interventions, has a significant positive clinical potential in victims of polytrauma.

Limitations in research. Financial resources, method and data collection.

Prospects for further research. Further clinical evaluation of the effect of autologous plasma with high platelet content in the optimization of reparative osteogenesis on the long-term prognosis in patients with combined pelvic trauma and multiple bone injuries is promising.

Conflicts of interest. Neither author has actual or potential conflicts of interest.

\section{References}

1. Von Rüden C, Bühren V, Perl M. Polytraumamanagement - Behandlung des Schwerverletzten in Schockraum und OP [Polytrauma Management - Treatment of Severely Injured Patients in ER and OR]. Z Orthop Unfall. 2017 Oct;155(5):603-622. German. doi: 10.1055/s-0042-124275. Epub 2017 Oct 19. PMID: 29050055.

2. Mann SM, Banaszek D, Lajkosz K, Brogly SB, Stanojev SM, Evans C, Bardana DD, Yach J, Hall S. High-energy trauma patients with pelvic fractures: Management trends in Ontario, Canada. Injury. 2018 Oct;49(10):1830-1840. doi: 10.1016/j.injury.2018.06.044. Epub 2018 Jul 6. PMID: 29997027. 
3. Caillot M, Hammad E, Le Baron M, Villes V, Leone M, Flecher X. Pelvic fracture in multiple trauma: A 67-case series. Orthop Traumatol Surg Res. 2016 Dec;102(8):1013-1016. doi: 10.1016/j.otsr.2016.08.018. Epub 2016 Nov 15. PMID: 27863917.

4. Toimela J, Brinck T, Handolin L. Evolution of high-energy pelvic trauma in southern Finland: a 12-year experience from a tertiary trauma centre. Eur J Trauma Emerg Surg. 2019 Sep 19. doi: 10.1007/s00068-019-01210-5. Epub ahead of print. PMID: 31538218.

5. Hsu SD, Chen CJ, Chou YC, Wang SH, Chan DC. Effect of Early Pelvic Binder Use in the Emergency Management of Suspected Pelvic Trauma: A Retrospective Cohort Study. Int J Environ Res Public Health. 2017 Oct 12;14(10):1217. doi: 10.3390/ijerph14101217. PMID: 29023379; PMCID: PMC5664718.

6. Höch A, Zeidler S, Pieroh P, Josten C, Stuby FM, Herath SC; German Pelvic Trauma Registry. Trends and efficacy of external emergency stabilization of pelvic ring fractures: results from the German Pelvic Trauma Registry. Eur J Trauma Emerg Surg. 2019 May 22. doi: 10.1007/s00068-019-01155-9. Epub ahead of print. PMID: 31119322.

7. Almahmoud K, Pfeifer R, Al-Kofahi K, Hmedat A, Hyderabad W, Hildebrand F, Peitzman AB, Pape HC. Impact of pelvic fractures on the early clinical outcomes of severely injured trauma patients. Eur J Trauma Emerg Surg. 2018 Apr;44(2):155-162. doi: 10.1007/s00068-016-0754-6. Epub 2017 Jan 13. PMID: 28091737.

8. Rossi LA, Murray IR, Chu CR, Muschler GF, Rodeo SA, Piuzzi NS. Classification systems for platelet-rich plasma. Bone Joint J. 2019 Aug;101-B(8):891-896. doi: 10.1302/0301-620X.101B8.BJJ-2019-0037.R1. PMID: 31362555.

9. Huang G, Hua S, Yang T, Ma J, Yu W, Chen X. Platelet-rich plasma shows beneficial effects for patients with knee osteoarthritis by suppressing inflammatory factors. Exp Ther Med. 2018 Mar;15(3):3096-3102. doi: 10.3892/etm.2018.5794. Epub 2018 Jan 24. PMID: 29599843; PMCID: PMC5867452.

10. Kapse S, Surana S, Satish M, Hussain SE, Vyas S, Thakur D. Autologous plateletrich fibrin: can it secure a better healing? Oral Surg Oral Med Oral Pathol Oral Radiol. 2019 Jan;127(1):8-18. doi: 10.1016/j.oooo.2018.08.010. Epub 2018 Aug 31. PMID: 30287202.

11. Khatab S, van Buul GM, Kops N, Bastiaansen-Jenniskens YM, Bos PK, Verhaar JA, van Osch GJ. Intra-articular Injections of Platelet-Rich Plasma Releasate Reduce Pain and Synovial Inflammation in a Mouse Model of Osteoarthritis. Am J Sports Med. 2018 Mar;46(4):977-986. doi: 10.1177/0363546517750635. Epub 2018 Jan 26. PMID: 29373806.

12. Tischer T, Bode G, Buhs M, Marquass B, Nehrer S, Vogt S, Zinser W, Angele P, Spahn G, Welsch GH, Niemeyer P, Madry H. Platelet-rich plasma (PRP) as therapy for cartilage, 
tendon and muscle damage - German working group position statement. J Exp Orthop. 2020 Sep 3;7(1):64. doi: 10.1186/s40634-020-00282-2. PMID: 32885339; PMCID: PMC7471237.

13. Le ADK, Enweze L, DeBaun MR, Dragoo JL. Platelet-Rich Plasma. Clin Sports Med. 2019 Jan;38(1):17-44. doi: 10.1016/j.csm.2018.08.001. PMID: 30466721.

14. Castillo-Cardiel G, Medina-Quintana VM, Lomelí-Enríquez M. Plasma rico en plaquetas y su efecto en la regeneración ósea en fracturas mandibulares. Ensayo clínico controlado [Platelet-rich plasma and its effect in bone regeneration in mandibular fractures. Controlled clinical trial]. Gac Med Mex. 2017;153(4):459-465. Spanish. doi: 10.24875/GMM.17002574. PMID: 28991280.

15. Szponder T, Wessely-Szponder J, Sobczyńska-Rak A, Żylińska B, Radzki RP, Polkowska I. Application of Platelet-rich Plasma and Tricalcium Phosphate in the Treatment of Comminuted Fractures in Animals. In Vivo. 2018 Nov-Dec;32(6):1449-1455. doi: 10.21873/invivo.11398. PMID: 30348700; PMCID: PMC6365725.

16. Gunay S, Candan H, Y1lmaz R, Eser I, Aydoğmus U. The Efficacy of PlateletRich Plasma in the Treatment of Rib Fractures. Thorac Cardiovasc Surg. 2017 Oct;65(7):546-550. doi: 10.1055/s-0036-1582261. Epub 2016 May 5. PMID: 27148928.

17. Singh R, Rohilla R, Gawande J, Kumar Sehgal P. To evaluate the role of plateletrich plasma in healing of acute diaphyseal fractures of the femur. Chin J Traumatol. 2017 Feb;20(1):39-44. doi: 10.1016/j.cjtee.2016.03.007. Epub 2017 Jan 20. PMID: 28202370; PMCID: PMC5343097.

18. Wang D, Rodeo SA. Platelet-Rich Plasma in Orthopaedic Surgery: A Critical Analysis Review. JBJS Rev. 2017 Sep;5(9):e7. doi: 10.2106/JBJS.RVW.17.00024. PMID: 28953136.

19. Cheung EC, Hodax JD, Hsu WK, Williams SK, Smith HE, Lansdown DA, Feeley BT. Platelet-Rich Plasma, Bone Morphogenetic Protein, and Stem Cell Therapies. Instr Course Lect. 2020;69:273-288. PMID: 32017733.

20. Li SY, Zhou QS, Li Y, Chen C, Chen JQ, Zhou HL. [Autologous platelet-rich plasma combined with bone grafting in inducing membrane technology]. Zhongguo Gu Shang. 2019 Apr 25;32(4):302-307. Chinese. doi: 10.3969/j.issn.1003-0034.2019.04.003. PMID: 31027404.

21. Acosta-Olivo C, Garza-Borjon A, Simental-Mendia M, Vilchez-Cavazos F, Tamez-Mata Y, Peña-Martinez V. Delayed union of humeral shaft fractures: comparison of autograft with and without platelet-rich plasma treatment: a randomized, single blinded clinical 
trial. Arch Orthop Trauma Surg. 2017 Sep;137(9):1247-1252. doi: 10.1007/s00402-017-2736-5. Epub 2017 Jun 30. PMID: 28667397.

22. Canbeyli İD, Akgun RC, Sahin O, Terzi A, Tuncay İC. Platelet-rich plasma decreases fibroblastic activity and woven bone formation with no significant immunohistochemical effect on long-bone healing: An experimental animal study with radiological outcomes. J Orthop Surg (Hong Kong). 2018 May-Aug;26(3):2309499018802491. doi: 10.1177/2309499018802491. PMID: 30295139.

23. Leitner L, Gruber G, Lohberger B, Kaltenegger H, Leithner A, Sadoghi P. Klinische Anwendung von Platelet-rich plasma und Wachstumsfaktoren am Bewegungsapparat [Clinical administration of platelet-rich plasma and growth factors to the musculoskeletal system]. Orthopade. 2019 Jan;48(1):105-116. German. doi: 10.1007/s00132-018-3643-3. PMID: 30259059. 